\title{
Formation of Virus-like Clusters Is an Intrinsic Property of the Tumor Necrosis Factor Family Member BAFF (B Cell Activating Factor) ${ }^{\dagger}$
}

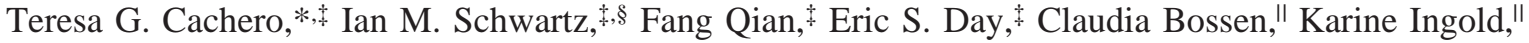 \\ Aubry Tardivel," Dennis Krushinskie, $₫$ John Eldredge,, Laura Silvian, ${ }^{\ddagger}$ Alexey Lugovskoy, ${ }^{\ddagger}$ Graham K. Farrington,, \\ Kathy Strauch, Pascal Schneider," and Adrian Whitty \\ Biogen Idec, Inc., 14 Cambridge Center, Cambridge, Massachusetts 02142, and Department of Biochemistry, University of \\ Lausanne, CH-1066 Epalinges, Switzerland
}

Received August 24, 2005; Revised Manuscript Received December 6, 2005

\begin{abstract}
The oligomeric state of BAFF (B cell activing factor), a tumor necrosis factor (TNF) family cytokine that plays a critical role in B cell development and survival, has been the subject of recent debate. Myc-tagged BAFF starting at residue Gln136 was previously reported to crystallize as trimers at $\mathrm{pH} 4.5$, whereas a histidine-tagged construct of BAFF, starting at residue Ala134, formed a virus-like cluster containing 60 monomers when crystallized at $\mathrm{pH}$ 9.0. The formation of the BAFF 60-mer was $\mathrm{pH}$ dependent, requiring $\mathrm{pH} \geq 7.0$. More recently, 60-mer formation was suggested to be artificially induced by the histidine tag, and it was proposed that BAFF, like all other TNF family members, is trimeric. We report here that a construct of BAFF with no amino-terminal tag (Ala134-BAFF) can form a 60-mer in solution. Using size exclusion chromatography and static light scattering to monitor trimer to 60-mer ratios in BAFF preparations, we find that 60 -mer formation is $\mathrm{pH}$-dependent and requires histidine 218 within the DE loop of BAFF. Biacore measurements established that the affinity of Ala134-BAFF for the BAFF receptor BAFFR/BR3 is similar to that of myc-Gln136-BAFF, which is exclusively trimeric in solution. However, Ala134-BAFF is more efficacious than myc-Gln136-BAFF in inducing B cell proliferation in vitro. We additionally show that BAFF that is processed and secreted by $293 \mathrm{~T}$ cells transfected with full-length BAFF, or by a histiocytic lymphoma cell line (U937) that expresses BAFF endogenously, forms a $\mathrm{pH}$-dependent 60 -mer in solution. Our results indicate that the formation of the 60-mer in solution by the BAFF extracellular domain is an intrinsic property of the protein, and therefore that this more active form of BAFF may be physiologically relevant.
\end{abstract}

$\mathrm{BAFF}^{1}$ (B cell activating factor), also known as BLyS, TALL-1, THANK, and zTNF4, is a protein belonging to the tumor necrosis factor (TNF) family of cytokines that is critical for the survival of B cells. It is expressed in macrophages, monocytes, dendritic cells, neutrophils, to a lesser extent, $\mathrm{T}$ cells, and other nonhematopoetic cells (reviewed in ref 1 ). BAFF is a type II transmembrane protein that can be proteolytically cleaved between $\operatorname{Arg} 133$ and

\footnotetext{
† This work was supported in part by grants from the Swiss National Science Fundation, including a grant from the NCCR (National Center of Competence in Research) Molecular Oncology to P.S.

* To whom correspondence should be addressed at Altus Pharmaceuticals, Inc., 625 Putnam Ave., Cambridge, MA 02139. E-mail: tcachero@altus.com. Phone: (617) 299-2885. Fax: (617) 299-2950.

Biogen Idec, Inc.

$\S$ Current address: ImmunoGen, Inc., 128 Sidney St., Cambridge, MA 02139.

"University of Lausanne.

${ }^{1}$ Abbreviations: APRIL, a proliferation-inducing ligand; BAFF, B cell activating factor of the TNF family; BAFFR, BAFF receptor; $\mathrm{BCMA}, \mathrm{B}$ cell maturation antigen; $\mathrm{BCMA}-\mathrm{Fc}$, fusion protein consisting of the extracellular domain of BCMA and the $\mathrm{C}_{\mathrm{H}} 1, \mathrm{C}_{\mathrm{H}} 2$, and hinge region of human IgG1; FBS, fetal bovine serum; GF-LS, gel filtration with in-line light scattering; PBS, phosphate-buffered saline $(20 \mathrm{mM}$ sodium phosphate, $\mathrm{pH} 7.4,140 \mathrm{mM} \mathrm{NaCl}$ ); SEC, size exclusion chromatography; TACI, transmembrane activator and CAML (calciummodulator and cyclophilin ligand) interactor; TNF, tumor necrosis factor.
}

Ala134 and released as a soluble protein $(2,3)$. BAFF knockout mice lack mature B cells in the periphery, showing that BAFF is required for B cell development in vivo $(4,5)$. Mice overexpressing BAFF display symptoms of autoimmune disorders (6). Moreover, elevated levels of soluble BAFF are detected in the blood of patients with various autoimmune diseases $(7-10)$. Interestingly, BAFF has also been reported to form biologically active heteromers with APRIL (a proliferation-inducing ligand), a closely related TNF family ligand. These heterotrimers have been detected in serum samples from patients with systemic immune-based rheumatic diseases (11).

BAFF can signal through three receptors: B cell maturation antigen (BCMA), transmembrane activator and cyclophilin ligand interactor (TACI), and BAFF receptor (BAFFR, BR3). BCMA and TACI also bind APRIL (reviewed in ref 1). BAFF costimulates the proliferation of $\mathrm{B}$ cells in the presence of anti-IgM antibody (3), and fusion proteins of the BAFF receptors with the $\mathrm{C}_{\mathrm{H}} 1, \mathrm{C}_{\mathrm{H}} 2$, and hinge region of human $\operatorname{IgG1}$ were shown to block this activity in vitro (4, $7,12,13)$. BAFFR is expressed on all peripheral B cells beyond the transitional type I stage of development and is the specific receptor for $\operatorname{BAFF}(13,14)$. Mice lacking BAFFR have a phenotype similar to that of BAFF knockout mice $(13,14)$. Recently, studies with monomeric receptors 
have shown that BAFF binds BAFFR with 100-fold higher affinity than it binds BCMA (15).

All other TNF family ligands exist as trimers. We have previously reported the crystal structure of the BAFF extracellular domain, using a construct starting at residue Gln136 and with an amino-terminal myc tag (myc-Q136BAFF). The structure, obtained at $\mathrm{pH} 4.5$, showed two BAFF trimers per asymmetric unit. Like other TNF family members, each monomer of BAFF folds as a sandwich of two antiparallel $\beta$-sheets. The structure of BAFF revealed that the loop connecting $\beta$ strands $\mathrm{D}$ and $\mathrm{E}$ is longer than the corresponding loops seen in other TNF family members (16). Oren and co-workers have independently reported the crystal structure of BAFF at pH 6.0, which also showed a dimer of trimers in the asymmetric unit (17). Interestingly, Liu et al. (18) reported that a BAFF construct starting at residue Ala134 and with an N-terminal histidine tag (His-A134BAFF), when crystallized at $\mathrm{pH} 9.0$, displayed an oligomeric, virus-like structure containing 20 trimers (60 monomers, 60mer). It appeared that the interaction between trimers was mediated by the long DE loop. The authors showed that formation of the BAFF 60-mer in solution was pH-dependent, and was abolished by deletion of the DE loop. They proposed that the ionization state of histidine 218 in the DE loop is responsible for the $\mathrm{pH}$ dependence of 60-mer formation (18). The crystal structures of the BAFF-BAFFR and BAFF-BCMA complexes have been solved and show a similar, virus-like BAFF 60-mer, with each of the 60 receptor binding sites occupied by a receptor molecule (19). Recently, a report by Zhukovsky and colleagues questioned the relevance of oligomeric BAFF in solution (20). They suggested that BAFF, like other TNF family members, exists as a trimer and that the 60-mer formation reported by Liu et al. (18) was an artifact of the histidine tag present in their construct. Furthermore, Zhukovsky and co-workers reported that histidine-tagged and untagged BAFF protein showed equivalent activity in a cell-based assay, from which they concluded that 60 -mer formation did not alter the functional activity of the protein.

To characterize the ability of BAFF to form 60-mers in solution and the consequences for functional activity, we engineered a construct of BAFF comprising the TNF homologous domain (Ala134-Leu285) with no aminoterminal tag (A134-BAFF). Since A134-BAFF purified from Pichia is glycosylated, we also engineered an aglycosylated version, A134-BAFF-N242Q. We report here that both A134-BAFF and A134-BAFF-N242Q form 60-mers in solution in a pH-dependent manner and that 60 -mer formation is abolished by the mutation of histidine 218 within the DE loop to alanine. We also show that the affinity of A134BAFF for BAFFR is identical to that of myc-Q136-BAFF, which we showed to be exclusively trimeric in solution, but that A134-BAFF shows higher activity than myc-Q136$\mathrm{BAFF}$ in a cell-based assay measuring costimulation of B cell proliferation. Taken together, our results indicate that BAFF 60-mer formation does not require an $\mathrm{N}$-terminal histidine tag, but rather is an intrinsic property of the BAFF protein. We additionally show that significant levels of the BAFF 60-mer are detected in culture supernatants when BAFF is naturally processed and secreted after transient transfection into 293T cells, and also when BAFF is endogenously expressed in U937 cells. These results support the notion that the BAFF 60-mer is not just an in vitro experimental artifact, but that this more active form of the protein could have a physiological role.

\section{MATERIALS AND METHODS}

Expression of BAFF Proteins. All protein expression was in Pichia pastoris GS115 using the methanol-inducible native AOX1 promoter, or the GS115 derivative MMC216 using the doxycycline-inducible TetO-AOX1 promoter (M. Levesque, D. Krushinskie, and K. Strauch, manuscript in preparation). Expression plasmids used the $\alpha$ factor secretion signal and the HIS4 selectable marker. Transformation was by electroporation or the P. pastoris EasyComp kit (Invitrogen). Manipulation and strain construction methods were as recommended by Invitrogen. StuI digestion was used to linearize DNA prior to transformation. The N242Q and H218A mutations were constructed by QuikChange (Stratagene) site-directed mutagenesis. Proteins were expressed by shake flask induction in BMGY and BMMY $(2 \% \mathrm{MeOH})$ according to Invitrogen recommendations for Myc-BAFF, or by fermentation in a reduced salts basal salts hexametaphosphate medium (M. Levesque, D. Krushinskie, and K. Strauch, manuscript in preparation) for A134-BAFF (A134L285), A134-BAFF-H218A, His-A134-BAFF, and A134BAFF-N242Q. Induction in fermenters was achieved with $1 \mu \mathrm{g} / \mathrm{mL}$ doxycyline or by $\mathrm{MeOH}$ fed-batch growth.

Purification of BAFF Proteins. The following proteins were expressed in P. pastoris: myc-Q136-BAFF (Q136L285), A134-BAFF (A134-L285), A134-BAFF-H218A, A134-BAFF-N242Q, and His-A134-BAFF. The purification of myc-BAFF was as reported (15). Briefly, P. pastoris supernatants were diafiltered by tangential flow filtration (Millipore, Pellicon II) and purified using ion exchange chromatography (Q XL Sepharose, Q HP Sepharose, and SP Sepharose; Amersham Biosciences). Purified proteins were dialyzed against $10 \mathrm{mM}$ Tris, $\mathrm{pH} 7.5,150 \mathrm{mM} \mathrm{NaCl}$. His-A134-BAFF was purified on a nickel column (Amersham Biosciences). Proteins were over $90 \%$ pure as confirmed by SDS-PAGE, and mass spectrometry analyses confirmed that the proteins had the expected molecular mass.

Analytical Gel Filtration of Purified BAFF. BAFF proteins were dialyzed for $3 \mathrm{~h}$ or overnight against the following buffers containing $150 \mathrm{mM} \mathrm{NaCl}$ : pH $5.0(25 \mathrm{mM}$ sodium acetate), pH 7.5 (10 mM Tris-HCl), pH $8.0(20 \mathrm{mM}$ Tris$\mathrm{HCl})$, and $\mathrm{pH} 9.0(20 \mathrm{mM}$ Tris $-\mathrm{HCl})$. Proteins $(0.5-1 \mathrm{mg})$ were then analyzed in a Superdex 200 10/30 tricorn column (Amersham Biosciences) in the appropriate buffer. In some cases, peak fractions were subsequently analyzed by Western blotting using a rabbit polyclonal antibody raised against the carboxy terminus of BAFF (Chemicon). In some instances, peak fractions were concentrated 5-fold using Microcon YM30 (Amicon, Millipore, $30000 \mathrm{MWCO}$ ) prior to Western blot.

Gel Filtration Assays with Light Scattering. Size exclusion chromatography (SEC) was carried out on a YMC-Pack Diol120 column, $8.0 \times 300 \mathrm{~mm}$ (YMC, Inc., Wilmington, NC), in $20 \mathrm{mM}$ sodium phosphate buffer, $\mathrm{pH} 7.2,150 \mathrm{mM} \mathrm{NaCl}$ (PBS) using a flow rate of $0.6 \mathrm{~mL} / \mathrm{min}$ on a Waters Alliance instrument (Waters, Milford, MA). In addition to UV detection, the eluent was monitored in tandem with a refractive index detector (Waters) and a Precision Detector 
PD2000 light scattering instrument (Precision Detectors, Bellingham, MA). Static light scattering was measured on a Precision Detector PD2000/DLS instrument equipped with a dual-angle flow cell detector. Molecular weight determination of each complex was performed with the Precision Detector software.

Western Blots. The rabbit anti-human BAFF polyclonal antibody was purchased from Upstate.

The ARH-77 plasma cell leukemia, Hut-78 cutaneous T lymphoma, K562 chronic myelogenous leukemia, U266 myeloma, RPMI8226 myeloma, Molt-4 T acute lymphoblastic leukemia, SKW6.4 B lymphoblast, U937 histiocytic lymphoma, L428 Hodgkin's lymphoma, THP-1 acute monocytic leukemia, L363 plasma cell leukemia, BL41 Burkitt's lymphoma, OPM-2 myeloma, L428 Hodgkin's lymphoma, and HL-60 promyelocytic leukemia cell lines were obtained from various collaborators and from the ATCC.

Immunoprecipitations. A $500 \mu \mathrm{L}$ sample of cell supernatants was mixed with $1 \mu \mathrm{g} / \mathrm{mL}$ hBCMA-Fc (Axxora, San Diego) and immunoprecipitated with $5 \mu \mathrm{L}$ of Protein A-Sepharose. Beads were recovered in microcolumns, washed with $3 \times 200 \mu \mathrm{L}$ of PBS, and eluted with $15 \mu \mathrm{L}$ of $0.1 \mathrm{M}$ citrate- $\mathrm{NaOH}, \mathrm{pH}$ 2.7. The eluate was neutralized with 1 M Tris- $\mathrm{HCl}, \mathrm{pH} 9$, and analyzed by Western blotting.

Analytical Gel Filtration of BAFF in Cell Supernatants. 293 T cells were transfected with a mammalian expression vector for full-length, untagged hBAFF (3) and grown for 3 days in serum-free OptiMEM-1 medium (Invitrogen) supplemented with $10 \mathrm{mM}$ Hepes, $\mathrm{pH} 7.4$. U937 cells were grown for 11 days in RPMI medium supplemented with $10 \%$ fetal bovine serum and $0.1 \%$ sodium bicarbonate. Conditioned supernatants were concentrated 10-fold in an Amicon Ultra centrifugal filtration device with a molecular weight cutoff of 10000 , and loaded (200 $\mu \mathrm{L})$ onto a Superdex 200 10/30 column equilibrated in either PBS, $\mathrm{pH} 7.4$, or $25 \mathrm{mM}$ sodium acetate, $150 \mathrm{mM} \mathrm{NaCl}, \mathrm{pH}$ 5. The column was eluted at 0.5 $\mathrm{mL} / \mathrm{min}$, and fractions of $1.5 \mathrm{~mL}$ were collected. In the case of U937 supernatants, $430 \mu \mathrm{L}$ of the fractions was supplemented with $20 \mu \mathrm{g}$ of bovine serum albumin and precipitated for $10 \mathrm{~min}$ on ice with trichloroacetic acid at a final concentration of $5 \%$. After centrifugation, the supernatants were carefully discarded, and the pellets were resuspended by sonication in $60 \mu \mathrm{L}$ of reducing SDS-PAGE sample buffer. BAFF was detected by Western blotting with a rabbit anti-BAFF polyclonal antibody (Upstate) and ECL reagent (Amersham) or, for the Superdex elution of U937 supernatants, with SuperWest femto ECL reagent (Pierce).

Solution-Phase Affinity Measurements. All experiments were performed on a Biacore 3000 instrument (Biacore AB, Uppsala, Sweden). Chip preparation and solution-phase binding measurements have been described (15). Briefly, monomeric BAFFR and the various forms of BAFF were mixed in different ratios in Biacore assay buffer $(10 \mathrm{mM}$ HEPES, pH 7.0, $150 \mathrm{mM} \mathrm{NaCl}, 3.4 \mathrm{mM}$ EDTA, $0.005 \%$ P-20 detergent, $0.1 \%$ BSA) and preincubated for a minimum of $3 \mathrm{~h}$ at $4{ }^{\circ} \mathrm{C}$ to reach equilibrium. The equilibrated solutions were then injected over a BCMA-Fc-derivatized surface, and the concentration of free BAFF in solution was measured. The affinity of the interaction of BAFFR with various forms of BAFF was determined from a plot of the concentration of free BAFF versus total soluble receptor by fitting the data to a quadratic binding equation as described (15).

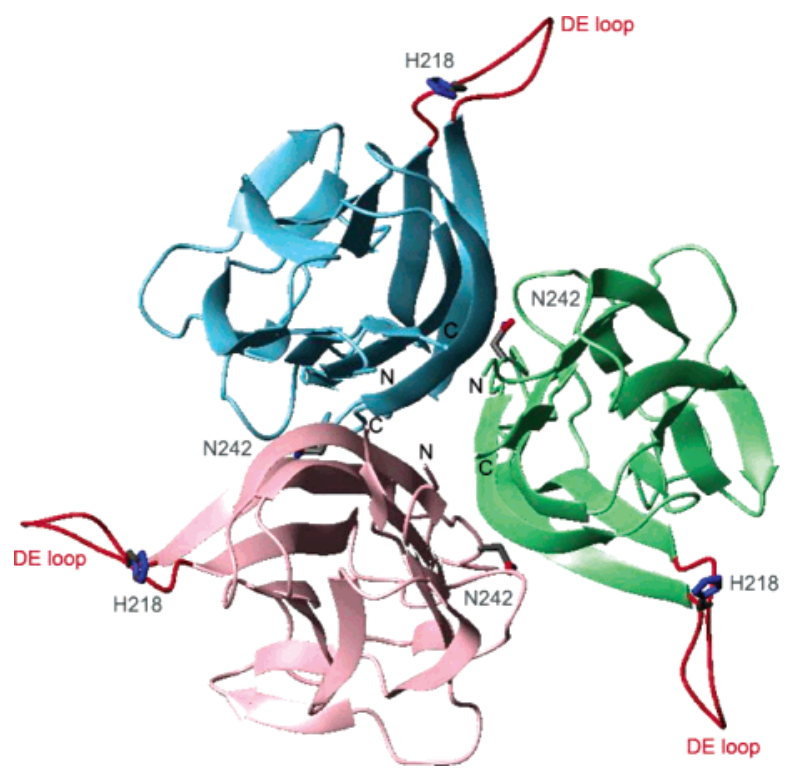

FIGURE 1: Crystal structure of BAFF. The extracellular domain of human BAFF (PDB ID 1kd7; 16) is shown in ribbon representation. The three chains are colored in cyan, green, and pink. The DE loops are colored in red. Side chains of amino acids N242 and H218 are shown in stick representation.

B Cell Proliferation Assay. The proliferation assay has been described $(13,15)$. Briefly, B cells isolated from mouse splenocytes were incubated with $2 \mu \mathrm{g} / \mathrm{mL}$ goat anti-mouse IgM antibody and different concentrations of BAFF for 48 h. Cells were pulsed for an additional $18 \mathrm{~h}$ with $\left[{ }^{3} \mathrm{H}\right]-$ thymidine (Amersham Biosciences) $(1 \mu \mathrm{Ci} /$ well $)$ and harvested. $\left[{ }^{3} \mathrm{H}\right]$ Thymidine incorporation was monitored by liquid scintillation counting.

\section{RESULTS}

To test whether the formation of the BAFF 60-mer is dependent on the presence of an N-terminal histidine tag as has been proposed (20), we engineered a BAFF construct starting at amino acid alanine 134 with no amino-terminal tag. This construct is similar to that reported by Liu and coworkers (18), but lacking the histidine tag (Figure 1). Recombinant BAFF purified from $P$. pastoris is glycosylated at amino acid asparagine 242 (16). It has been shown that soluble human BAFF expressed in 293T cells is not glycosylated (3). Moreover, the construct reported by Liu and co-workers was purified from Escherichia coli, and hence was not glycosylated. Therefore, we also prepared a second construct in which residue 242 was mutated to glutamine (N242Q) to eliminate the N-linked glycosylation site and thus ensure that our yeast-expressed protein was not glycosylated. We refer to this protein as A134-BAFF-N242Q.

We evaluated the oligomeric state of both A134-BAFF and A134-BAFF-N242Q in solution by analytical gel filtration at $\mathrm{pH}$ 7.5. When $5.8 \mu \mathrm{M}$ Ala134-BAFF protein (molarity based on the BAFF monomer) was loaded onto a gel filtration column, the protein eluted as an oligomer (MW > 670000 ) with a small portion eluting as a trimer (Figure 2). To test how the formation of the 60-mer varies with protein concentration, we analyzed the elution profile of A134-BAFF at decreasing concentrations, and detected BAFF in peak fractions by Western blot analysis. A134-BAFF eluted as a 60 -mer at all tested concentrations, the lowest being $14 \mathrm{nM}$ 


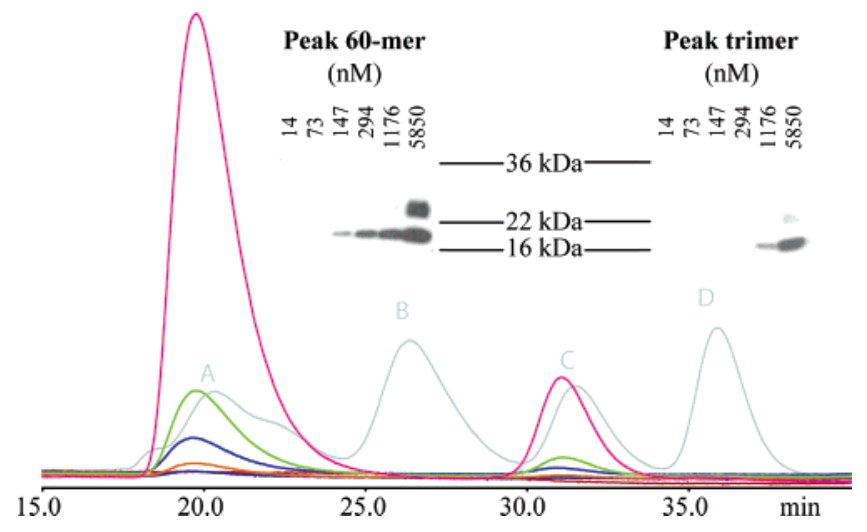

FIGURE 2: A134-BAFF forms a 60-mer in solution. Size exclusion chromatography and Western blot analysis of A134-BAFF. SEC was carried out as described in the Materials and Methods. Molecular weight standards (A280, gray line) are as follows: A, thyroglobulin, $\mathrm{MW}=670000 ; \mathrm{B}, \gamma$ globulin, $\mathrm{MW}=158000 ; \mathrm{C}$, ovalbumin, $\mathrm{MW}=44$ 000; $\mathrm{D}$, myoglobulin, $\mathrm{MW}=17$ 000. A134BAFF was loaded onto the gel filtration column at the indicated concentrations, eluted at $0.5 \mathrm{~mL} / \mathrm{min}$, and collected in $500 \mu \mathrm{L}$ fractions (A214, colored lines). A134-BAFF concentrations (molarity based on the BAFF monomer) (nM) are 5850 (pink line), 1176 (green line), 294 (blue line), 147 (orange line), 73 (purple line), and 14 (red line). The inset plot shows the Western blot of the peak fractions. Molecular weight markers are shown between the panels. The 60-mer panel corresponds to the peak eluting at $\sim 20 \mathrm{~min}$, and the trimer panel corresponds to the peak eluting at $\sim 31 \mathrm{~min}$.

Table 1: Measurement of the Molecular Weight of BAFF Proteins by Gel Filtration Coupled to Light Scattering: Summary of Light Scattering Results

\begin{tabular}{lrc}
\hline \multicolumn{1}{c}{ protein } & $\begin{array}{c}\text { MW from LS, } \\
\pm 5 \%\end{array}$ & $\begin{array}{c}\text { theor MW } \\
\text { calcd from } \\
\text { the sequence }\end{array}$ \\
\hline A134-BAFF (60-mer) & 1060000 & 1022000 \\
A134-BAFF-N242Q (60-mer) & 960000 & 1023000 \\
His-A134-BAFF (60-mer) & 1020000 & 1090000 \\
Q136-BAFF (60-mer) & 1070000 & 1012000 \\
Myc-Q136-BAFF (trimer) & 55800 & 55600 \\
Myc-Q136-BAFF-N24 2Q (trimer) & 54600 & 55700 \\
A134-BAFF-H218A (trimer) & 56600 & 50900 \\
\hline
\end{tabular}

BAFF polypeptide corresponding to $245 \mathrm{pM} 60$-mer BAFF (Figure 2 and data not shown). Similar results were observed with Ala134-BAFF-N242Q, and with Ala134-BAFF that had been preincubated in cell culture media (RPMI, 10\% FBS) for 24 or $48 \mathrm{~h}$ at $37{ }^{\circ} \mathrm{C}$ (data not shown) to mimic the conditions used in subsequent cell-based assays. To directly measure the molecular weight of the BAFF oligomers in solution, we performed analytical gel filtration with in-line light scattering (GF-LS) at $\mathrm{pH} 7.2(21)$. Table 1 shows that both A134-BAFF-N242Q and A134-BAFF eluted with a molecular weight calculated from light scattering of 1060000 \pm 50000 . This value closely matches the molecular weight expected for the BAFF 60-mer (1 022 000). We also prepared a BAFF construct containing an $\mathrm{N}$-terminal histidine tag (His-A134-BAFF). In agreement with previous reports on histidine-tagged $\mathrm{BAFF}(18,20)$, this protein was also seen to exist as a 60-mer by GF-LS (Table 1). The observation that both A134-BAFF and His-A134-BAFF are able to form oligomers in solution shows that BAFF 60-mer formation does not require an $\mathrm{N}$-terminal histidine tag. In contrast, two constructs containing an N-terminal myc tag (myc-Q136BAFF-N242Q and myc-Q136) were shown to be exclusively
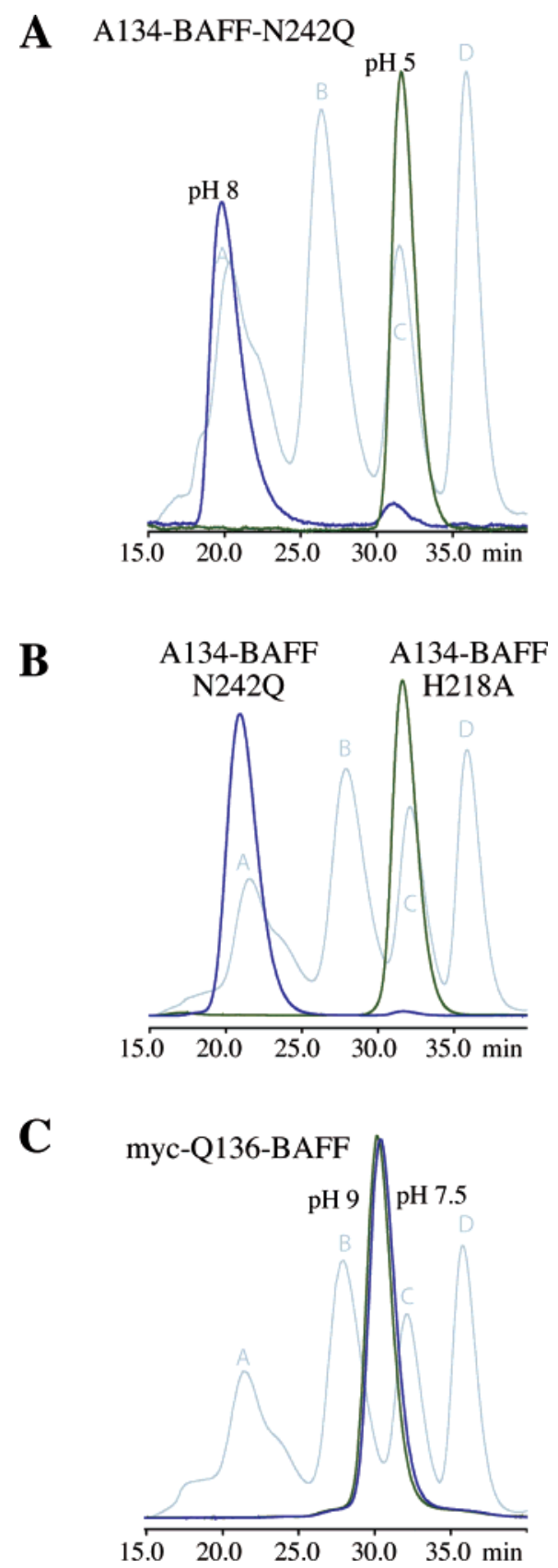

FIGURE 3: Characterization of BAFF proteins by analytical gel filtration. (A) $\mathrm{pH}$ dependence of 60-mer formation. A134-BAFF$\mathrm{N} 242 \mathrm{Q}$ was analyzed at $\mathrm{pH} 5.0$ (green) and $\mathrm{pH} 8.0$ (blue). Buffer conditions are described in the Materials and Methods. (B) H218A mutation abolishes 60-mer formation. A134-BAFF-N242Q (blue) and A134-BAFF-H218A (green) were analyzed in $10 \mathrm{mM}$ Tris, $\mathrm{pH} 7.5,150 \mathrm{mM} \mathrm{NaCl}$. (C) Myc-Q136-BAFF is trimeric even at high $\mathrm{pH}$. Myc-Q136-BAFF was characterized at $\mathrm{pH} 7.5$ (blue) and 9.0 (green). The molecular weight markers shown are as in Figure 2 (gray).

trimeric by analytical gel filtration and GF-LS with molecular weights from light scattering of $54600 \pm 3000$ and $55800 \pm 3000$ (Table 1).

To investigate the $\mathrm{pH}$ dependence of BAFF 60-mer formation, we dialyzed A134-BAFF-N242Q in a series of buffers with $\mathrm{pH}$ values ranging from 5.0 to 8.0. The oligomeric state of BAFF was then determined by analytical gel filtration as shown in Figure 3A. At $\mathrm{pH} 8.0$ very little trimeric BAFF was detected; the protein eluted largely as an oligomer with an apparent molecular weight greater than 
670000 . However, at pH 5.0, BAFF eluted as a trimer (Figure 3A). This result indicates that a form of purified BAFF that has no histidine tag at the amino terminus forms a 60 -mer in solution in a $\mathrm{pH}$-dependent manner and thus the $\mathrm{pH}$ dependence observed by Liu et al. (18) is not due to ionization of the histidine tag.

To study the role of histidine 218 in 60-mer formation, we engineered a construct in which histidine 218 was mutated to alanine, A134-BAFF-H218A. The purified protein was characterized by analytical gel filtration at $\mathrm{pH} 7.5$ (Figure $3 \mathrm{~B}$ ) and also at $\mathrm{pH} 5.0$ and 9.0 (data not shown). We found that, unlike A134-BAFF, A134-BAFF-H218A was trimeric in solution at all $\mathrm{pH}$ values tested (Figure $3 \mathrm{~B}$ ), suggesting that the mutation $\mathrm{H} 218 \mathrm{~A}$ abolished oligomer formation. This result extends the observation of Liu and co-workers (18), who noted that deletion of the entire DE loop disrupts 60mer formation, by showing that the same result can be achieved by this single-point mutation.

We also tested whether myc-Q136-BAFF, which is exclusively trimeric at $\mathrm{pH} 7.5$, can be induced to form a 60 mer at high $\mathrm{pH}$. To this end, we dialyzed the protein at $\mathrm{pH}$ 7.5 and 9.0 and analyzed its elution profile by analytical gel filtration. As shown in Figure 3C, myc-Q136-BAFF does not form a 60-mer even at high $\mathrm{pH}$.

We next wanted to investigate whether naturally processed full-length BAFF, and proteins produced by cell lines that express BAFF endogenously, form a 60-mer. Transfected $293 \mathrm{~T}$ cells have been shown to process full-length BAFF to its soluble form (3). 293T cells transfected with full-length human BAFF were grown in buffered culture medium to minimize acidification of the supernatants. The supernatant was shown by Western blot to contain secreted BAFF (data not shown), and the oligomeric state of this protein was analyzed by gel filtration. A significant fraction of the secreted BAFF eluted as an oligomer (MW $\geq 670000$ ), consistent with the presence of a BAFF 60-mer (Figure 4A). Identification of this fraction as a BAFF 60-mer was further supported by the $\mathrm{pH}$ dependence of this high molecular weight form of BAFF. In acidic conditions, the large oligomer dissociated entirely into trimers, as expected for the BAFF 60-mer (Figure 4A). To determine whether protein secreted from cells that express BAFF endogenously also exists as a 60-mer, we screened a number of cell lines for the presence of soluble BAFF in their supernatants. A reactive band of about 17000 molecular weight, which is the expected size for soluble BAFF, was readily detected in conditioned supernatants of U937 histiocytic lymphoma cells (Figure 4B). These cells have been described previously to express BAFF (22), and we confirmed the identity of the reactive protein by immunoprecipitation with BCMA-Fc (Figure 4C). U937 cells were grown in buffered culture medium to minimize acidification of the supernatants. Analysis of the supernatants by gel filtration again showed that a significant fraction of endogenous BAFF eluted as a high molecular weight oligomer that was sensitive to acid conditions (Figure 4D). Taken together, these results show that a naturally cleaved, soluble form of BAFF with a high molecular weight is detected in supernatants of cells that express full-length BAFF ectopically and cells that express BAFF endogenously. The size of this BAFF species and its dissociation properties in acidic conditions strongly suggest
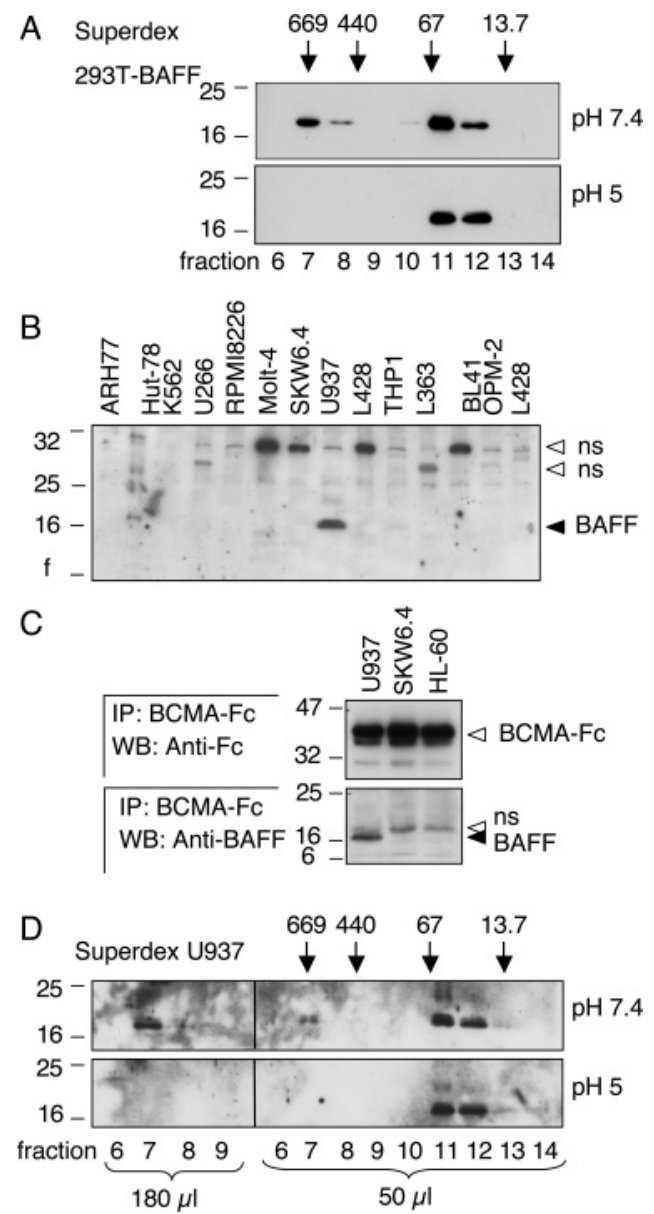

FIGURE 4: Endogenous BAFF forms pH-dependent 60-mers. (A) The $10 \times$ concentrated conditioned supernatants of $293 \mathrm{~T}$ cells transfected with full-length human BAFF were analyzed on a Superdex-200 column equilibrated at $\mathrm{pH} 7.4$ or 5. Samples of 20 $\mu \mathrm{L}$ out of $1.5 \mathrm{~mL}$ fractions were analyzed by Western blotting with a rabbit anti-BAFF antibody. The elution position of molecular weight markers is indicated at the top of the figure. (B) Samples of $20 \mu \mathrm{L}$ of conditioned supernatants from the indicated cell lines were analyzed by Western blotting using a polyclonal rabbit antiBAFF antibody. Bands indicated as "ns" result from nonspecific staining of unrelated proteins. (C) Cell supernatants of the indicated cell lines were mixed with BCMA-Fc, immunoprecipitated with Protein A-Sepharose, and analyzed by Western blotting using antiIg and anti-BAFF antibodies. (D) The $10 \times$ concentrated conditioned supernatants of U937 cells were analyzed on a Superdex-200 column as described above. Fractions were precipitated with $5 \%$ trichloroacetic acid, and an equivalent of 50 or $180 \mu \mathrm{L}$ of each fraction was analyzed by Western blotting using the anti-BAFF antibody. It was not possible to analyze $180 \mu \mathrm{L}$ of fractions 10 , 11 , and 12 because of the high quantity of bovine serum albumin contained in these fractions.

that naturally produced and processed BAFF can form 60 mers.

In the presence of anti-IgM antibody, BAFF costimulates the proliferation of B cells in vitro (3). Figure 5A shows that oligomeric A134-BAFF is more efficacious than trimeric myc-Q136-BAFF in inducing B cell proliferation in vitro. Interestingly, the mutation $\mathrm{H} 218 \mathrm{~A}$, which abolished 60-mer formation, resulted in activity that was identical to that of myc-Q136-BAFF (Figure 5A). Thus, myc-Q136-BAFF and A134-BAFF-H218A represent forms of BAFF that are unable to form a 60 -mer but retain biological activity.

To test whether the difference in functional activity observed between 60-mer and trimeric BAFF is due to a 

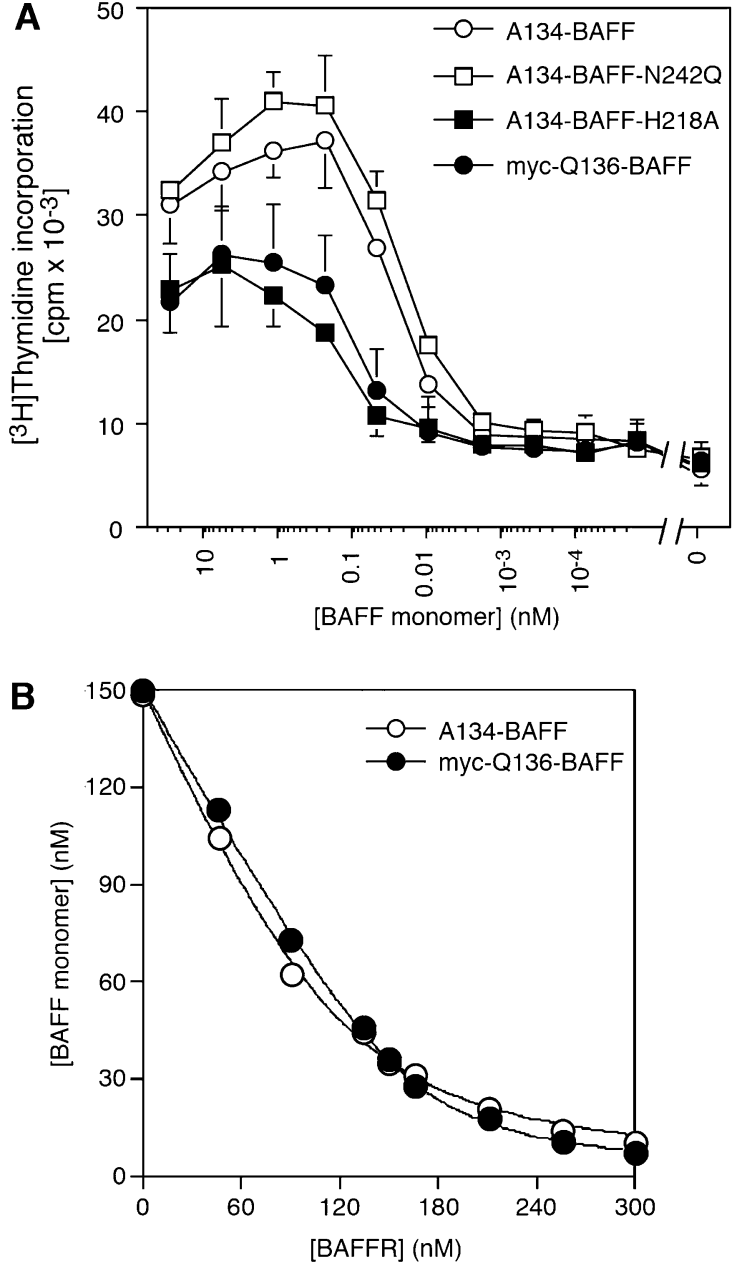

FIGURE 5: Functional activity of 60-mer versus trimeric BAFF. (A) Proliferation of purified splenic B cells induced by trimeric myc-Q136-BAFF (closed circles), trimeric A134-BAFF-H218A (closed squares), 60-mer A134-BAFF (open circles), and 60-mer A134-BAFF-N242Q (open squares). B cells were incubated in quadruplicate in the presence of goat anti-mouse $\operatorname{IgM}$ antibody and costimulated with increasing concentrations of BAFF. The data are representative of three independent experiments. (B) Affinity of myc-Q136-BAFF and A134-BAFF for monomeric BAFFR. The indicated concentrations of soluble, monomeric BAFFR were equilibrated in solution with a fixed concentration of BAFF (50 $\mathrm{nM}$ trimeric BAFF [myc-Q136, closed circles] or $2.5 \mathrm{nM} 60-\mathrm{mer}$ BAFF [A134-BAFF, open circles]). Solutions were then run over a BCMA-Fc-derivatized surface as described in the Materials and Methods. The affinity of the solution-phase binding of BAFFR with BAFF was determined by fitting the data to a quadratic binding equation as described (15).

difference in affinity for BAFFR, we evaluated the affinity of BAFF trimer and 60-mer binding to soluble monomeric BAFFR in solution using Biacore (Figure 5B). Titration of soluble BAFFR against either $50 \mathrm{nM}$ BAFF trimer or 2.5 nM BAFF 60-mer, equivalent to $150 \mathrm{nM}$ BAFF polypeptide in each case, shows that BAFFR binds to both forms of BAFF with similar affinities (15 and $9 \mathrm{nM}$, respectively). The affinity observed is in good agreement with previous results (15).

\section{DISCUSSION}

The solution structure of BAFF at physiological $\mathrm{pH}$ is a subject of debate. While some investigators believe that the physiological form of BAFF is a trimer like other TNF family ligands (20), others have reported that the active form of BAFF is a virus-like 60-mer containing 20 homotrimers (18). Formation of the BAFF 60-mer has been demonstrated by crystallography and in solution using a construct containing an amino-terminal histidine tag (18). In their cocrystallization of BAFF with BAFFR, Kim et al. used untagged A134BAFF which was coexpressed in insect cells with dimeric BAFFR-Fc (23). In this crystal structure BAFF appears as a 60-mer. However, they did not establish that this untagged BAFF can form a 60-mer in solution or in the absence of BAFFR-Fc. Zhukovsky et al. (20) observed that, of the three constructs His-A134-BAFF, Flag-A134-BAFF, and untagged A134-BAFF, only His-A134-BAFF formed 60-mers in solution. They concluded that the ability of BAFF to form 60 -mers is an artifact resulting from the presence of the histidine tag.

In contrast to the results of Zhukovsky et al. (20), we found by analytical gel filtration and light scattering that purified untagged glycosylated A134-BAFF or aglycosylated A134BAFF-N242Q proteins form 60-mers in solution. It is not clear why our results differ from those of Zhukovsky and co-workers. We used a different expression system, $P$. pastoris versus $E$. coli, and different buffer conditions, $\mathrm{pH}$ 7.5, no $\mathrm{MgCl}_{2}$ versus $\mathrm{pH} 8.0,25 \mathrm{mM} \mathrm{MgCl}$. To rule out buffer differences as a factor, we analyzed our untagged A134-BAFF-N242Q by analytical gel filtration using the same buffer conditions as Zhukovsky et al. and found that the protein still eluted as a 60-mer (data not shown).

The $\mathrm{pH}$ dependence of 60-mer formation by His-A134BAFF was ascribed, by Zhukovsky et al. (20), to the ionization state of the histidine tag, while Hong et al. (24) suggested that the $\mathrm{pH}$ dependence was instead due to the ionization of histidine 218 in the DE loop. The fact that our untagged BAFF shows the same $\mathrm{pH}$ dependence for 60-mer formation as that reported for the His-tagged protein indicates that the histidine tag is not solely responsible for this effect. Furthermore, our finding that mutating histidine 218 to alanine abolished 60-mer formation even at $\mathrm{pH} 8.0-9.0$ (Figure 3B, data not shown) is consistent with the $\mathrm{pH}$ dependence being due to ionization of histidine 218 .

BAFF is the only TNF family member that has an extended DE loop $(16,18)$. This region is involved in trimer-trimer interactions and was reported to be essential for BAFF activity though not for receptor binding (18). The importance of the DE loop region in the formation of the 60 -mer is underlined by the finding that murine APRIL, which lacks this long DE loop, is a trimeric protein at $\mathrm{pH}$ values ranging from 5 to 8.5 (25). Our observation that the $\mathrm{H} 218 \mathrm{~A}$ point mutation abolished 60 -mer formation is consistent with the role of the DE loop in 60-mer formation (18) and confirms that $\mathrm{H} 218$ in particular is important in mediating the interaction (24). However, in contrast to the findings of Liu et al., our less drastic mutational change allows us to separate the role of the DE loop in 60-mer formation from its role in the activity of BAFF. Our His218A construct, which gives rise exclusively to trimeric BAFF, was active in the $\mathrm{B}$ cell proliferation assay. Therefore, if the DE loop is required for functional activity, it is not solely due to its role in mediating 60-mer formation.

Our results indicate that an active form of BAFF, mycQ136-BAFF, is not able to form 60-mers in solution even at high $\mathrm{pH}$. To examine why myc-Q136-BAFF cannot form 
60-mers, we attempted to pack additional N-terminal amino acids of this construct inside the virus-like 60-mer structure reported previously (18) using protein structure modeling techniques $(26,27)$. The calculation (data not shown) suggested that the N-terminal extension of myc-Q136-BAFF is incompatible with virus-like assembly due to steric crowding, in contrast to the smaller His tag extension, providing a structural rationale for myc-Q136-BAFF being a trimer in solution. The fact that the N-terminal histidinetagged BAFF forms a 60-mer suggests that the energetic stabilization derived from trimer-trimer interaction mediated by the DE loop overcomes any electrostatic repulsion arising from the proximity of multiple histidine tags in the core of the 60-mer.

For applications where an exclusively trimeric BAFF protein is desired, myc-Q136-BAFF could be the construct of choice. Previous works that used this construct $(12,13$, 15,16 ) presumably reflect the properties of the BAFF trimer and are not influenced by the possibility of 60-mer formation. The A134-BAFF-H218A provides an alternative construct that it is solely trimeric and yet retains functional activity.

Having exclusively trimeric BAFF constructs in hand, we were able to compare the activities of the trimer versus the 60 -mer in receptor binding and functional assays. We find that the BAFF trimer and 60-mer bind monomeric BAFFR with similar affinities. This result is entirely consistent with the cocrystal structure of BAFF and BAFFR in that the crystal showed that the receptor binding sites on the BAFF 60-mer were fully accessible. There is, therefore, no structural reason to expect that the monovalent affinity of the receptor for the ligand would change between the trimer and 60-mer. The structural and binding data also argue against the existence of any significant allosteric cooperativity between the different receptor binding sites in the BAFF 60-mer.

Most TNF family ligands are type II membrane proteins, but many can be cleaved by metalloproteinases or furin-like convertases and released as soluble molecules. This is the case for TNF, Fas ligand (FasL), and BAFF, among others. Reports have shown that soluble FasL is less cytotoxic than membrane-bound FasL and that oligomerization of FasL is required to trigger activity $(28,29)$. Unlike trimeric soluble FasL, trimeric BAFF has been shown previously to be active and able to costimulate $\mathrm{B}$ cell proliferation in vitro (Figure $5 \mathrm{~A})(12,13)$. We reasoned that oligomeric BAFF could be more active in inducing $\mathrm{B}$ cell proliferation than trimeric BAFF due to the ability of 60-mer BAFF to engage more receptors at the plasma membrane, which in turn might induce a stronger signal. Our results indicate that the BAFF 60-mer is more active than the BAFF trimer in a B cell proliferation assay in vitro. Control experiments showed that the 60-mer remained intact when the protein was incubated in cell culture media (RPMI) at concentrations comparable to those used in this cell-based assay. The fact that the activity difference seen with the BAFF 60-mer is manifested in the amplitude of the response rather than its concentration dependence suggests that the increased multivalency of the 60 -mer affects the quality of the signal transduced rather than just the avidity of binding. Although 60-mer formation has not been demonstrated for murine BAFF, sequence alignment shows that the DE loop is conserved between the human and murine proteins. Mouse models may therefore be useful to test the physiological relevance of BAFF 60 -mers.
In conclusion, the ability of BAFF to form 60-mers in solution at physiological $\mathrm{pH}$ is an intrinsic property of this protein, and results in enhanced functional activity in vitro. Furthermore, our analysis of BAFF secreted by 293T cells transfected with full-length protein, and particularly by U937 cells that naturally express BAFF, shows that when expressed endogenously and processed naturally BAFF indeed forms 60-mers. These findings show that BAFF 60-mer formation is not restricted to truncated protein expressed ectopically, and therefore support the idea that the more active, 60-mer form of BAFF might occur in vivo and thus might have a physiological role.

\section{ACKNOWLEDGMENT}

We thank Azita Kaffashan, Craig Wildes, Dr. Chenhui Zeng, and Dr. Dingyi Wen for mass spectrometry analysis, Dr. Susan Kalled for reading the manuscript, and Dr. Blake Pepinsky for useful discussions.

\section{REFERENCES}

1. Kalled, S. L., Ambrose, C., and Hsu, Y. M. (2005) The biochemistry and biology of BAFF, APRIL and their receptors, Curr. Dir. Autoimmun. 8, 206-42.

2. Moore, P. A., Belvedere, O., Orr, A., Pieri, K., LaFleur, D. W., Feng, P., Soppet, D., Charters, M., Gentz, R., and Parmelee, D. (1999) BLyS: Member of the tumor necrosis factor family and B lymphocyte stimulator, Science 285, 260-263.

3. Schneider, P., MacKay, F., Steiner, V., Hofmann, K., Bodmer, J. L., Holler, N., Ambrose, C., Lawton, P., Bixler, S., Acha-Orbea, H., Valmori, D., Romero, P., Werner-Favre, C., Zubler, R. H., Browning, J. L., and Tschopp, J. (1999) BAFF, a novel ligand of the tumor necrosis factor family, stimulates B cell growth, J. Exp. Med. 189, 1747-56.

4. Gross, J. A., Dillon, S. R., Mudri, S., Johnston, J., Littau, A., Roque, R., Rixon, M., Schou, O., Foley, K. P., Haugen, H., McMillen, S., Waggie, K., Schreckhise, R. W., Shoemaker, K., Vu, T., Moore, M., Grossman, A., and Clegg, C. H. (2001) TACIIg neutralizes molecules critical for B cell development and autoimmune disease. Impaired B cell maturation in mice lacking BLyS, Immunity 15, 289-302.

5. Schiemann, B., Gommerman, J. L., Vora, K., Cachero, T. G., Shulga-Morskaya, S., Dobles, M., Frew, E., and Scott, M. L. (2001) An essencial role for BAFF in the normal development of B cells through a BCMA-independent pathway, Science 293, 2111-4.

6. Mackay, F., Woodcock, S. A., Lawton, P., Ambrose, C., Baetscher, M., Schneider, P., Tschopp, J., and Browning, J. L. (1999) Mice transgenic for BAFF develop lymphocyte disorders along with autoimmune manifestations, J. Exp. Med. 190, 1697-710.

7. Gross, J. A., Johnston, J., Mudri, S., Enselman, R., Dillon, S. R., Madden, K., Xu, W., Parrish-Novak, J., Foster, D., Lofton-Day, C., Moore, M., Littau, A., Grossman, A., Haugen, H., Foley, K., Blumberg, H., Harrison, K., Kindsvogel, W., and Clegg, C. H. (2000) TACI and BCMA are receptors for a TNF homologue implicated in B cell autoimmune disease, Nature 404, 995-9.

8. Groom, J., Kalled, S. L., Cutler, A. H., Olson, C., Woodcock, S. A., Schneider, P., Tschopp, J., Cachero, T. G., Batten, M., Wheway, J., Mauri, D., Cavill, D., Gordon, T. P., Mackay, C. R., and Mackay, F. (2002) Association of BAFF/BLyS overexpression and altered B cell differentiation with Sjogren's syndrome, J. Clin. Invest. 109, 59-68.

9. Zhang, J., Roschke, V., Baker, K. P., Wang, Z., Alarcon, G. S., Fessler, B. J., Bastian, H., Kimberly, R., and Zhou, T. (2001) Cutting edge: a role for B lymphocyte stimulator in systemic lupus erythematosus, J. Immunol. 166, 6-10.

10. Cheema, G. S., Roschke, V., Hilbert, D. M., and Stohl, W. (2001) Elevated serum B lymphocyte stimulator levels in patients with systemic immune-based rheumatic diseases, Arthritis Reum. 44, $1313-1319$.

11. Roschke, V., Sosnovtseva, S., Ward, C. D., Hong, J. S., Smith, R., Albert, V., Stohl, W., Baker, K. P., Ullrich, S., Nardelli, B., Hilbert, D. M., and Migone, T. S. (2002) BLyS and APRIL form 
biologically active heterotrimers that are expressed in patients with systemic immune-based rheumatic diseases, J. Immunol. 169, 4314-21.

12. Thompson, J. S., Schneider, P., Kalled, S. L., Wang, L., Lefevre, E. A., Cachero, T. G., MacKay, F., Bixler, S. A., Zafari, M., Liu, Z. Y., Woodcock, S. A., Qian, F., Batten, M., Madry, C., Richard, Y., Benjamin, C. D., Browning, J. L., Tsapis, A., Tschopp, J., and Ambrose, C. (2000) BAFF binds to the tumor necrosis factor receptor-like molecule $\mathrm{B}$ cell maturation antigen and is important for maintaining the peripheral B cell population, J. Exp. Med. 192, 129-35.

13. Thompson, J. S., Bixler, S. A., Qian, F., Vora, K., Scott, M. L., Cachero, T. G., Hession, C., Schneider, P., Sizing, I. D., Mullen, C., Strauch, K., Zafari, M., Benjamin, C. D., Tschopp, J., Browning, J. L., and Ambrose, C. (2001) BAFF-R, a newly identified TNF receptor that specifically interacts with BAFF, Science 293, 2108-11.

14. Yan, M., Brady, J. R., Chan, B., Lee, W. P., Hsu, B., Harless, S., Cancro, M., Grewal, I. S., and Dixit, V. M. (2001) Identification of a novel receptor for B lymphocyte stimulator that is mutated in a mouse strain with severe B cell deficiency, Curr. Biol. 11, $1547-52$

15. Day, E. S., Cachero, T. G., Qian, F., Sun, Y., Wen, D., Pelletier, M., Hsu, Y. M., and Whitty, A. (2005) Selectivity of BAFF/BLyS and APRIL for binding to the TNF family receptors BAFFR/BR3 and BCMA, Biochemistry 44, 1919-31.

16. Karpusas, M., Cachero, T. G., Qian, F., Boriack-Sjodin, A., Mullen, C., Strauch, K., Hsu, Y. M., and Kalled, S. L. (2002) Crystal structure of extracellular human BAFF, a TNF family member that stimulates B lymphocytes, J. Mol. Biol. 315, 1145-54.

17. Oren, D. A., Y., L., Volovik, Y., Morris, T. S., Dharia, C., Das, K., Galperina, O., Gentz, R., and Arnold, E. (2002) Structural basis of BLyS receptor recognition, Nat. Struct. Biol. 9, 288292.

18. Liu, Y., Xu, L., Opalka, N., Kappler, J., Shu, H. B., and Zhang, G. (2002) Crystal structure of sTALL-1 reveals a virus-like assembly of TNF family ligands, Cell 108, 383-94.

19. Liu, Y., Hong, X., Kappler, J., Jiang, L., Zhang, R., Xu, L., Pan, C. H., Martin, W. E., Murphy, R. C., Shu, H. B., Dai, S., and
Zhang, G. (2003) Ligand-receptor binding revealed by the TNF family member TALL-1, Nature 423, 49-56.

20. Zhukovsky, E. A., Lee, J. O., Villegas, M., Chan, C., Chu, S., and Mroske, C. (2004) TNF ligands: is TALL-1 a trimer or a virus-like cluster?, Nature 427, 413-4, discussion 414.

21. Wen, J., Arakawa, T., and Philo, J. (1996) Size-exclusion chromatography with on-line light-scattering, absorbance, and refractive index detectors for studying proteins and their interactions, Anal. Biochem. 240, 155-166.

22. Nardelli, B., Belvedere, O., Roschke, V., Moore, P. A., Olsen, H. S., Migone, T. S., Sosnovtseva, S., Carrell, J. A., Feng, P., Giri, J. G., and Hilbert, D. M. (2001) Synthesis and release of B-lymphocyte stimulator from myeloid cells, Blood 97, 198-204.

23. Kim, H. M., Yu, K. S., Lee, M. E., Shin, D. R., Kim, Y. S., Paik, S. G., Yoo, O. J., Lee, H., and Lee, J. O. (2003) Crystal structure of the BAFF-BAFF-R complex and its implications for receptor activation, Nat. Struct. Biol. 10, 342-8.

24. Hong, X., Kappler, J., Liu, Y., Xu, L., Shu, H. B., and Zhang, G. (2004) reply letter, Nature 427, 414.

25. Wallweber, H. J., Compaan, D. M., Starovasnik, M. A., and Hymowitz, S. G. (2004) The crystal structure of a proliferationinducing ligand, APRIL, J. Mol. Biol. 343, 283-90.

26. Sali, A., and Blundell, T. L. (1993) Comparative protein modelling by satisfaction of spatial restraints, J. Mol. Biol. 234, 779-815.

27. Fiser, A., Do, R. K., and Sali, A. (2000) Modeling of loops in protein structures, Protein Sci. 9, 1753-1773.

28. Schneider, P., Holler, N., Bodmer, J. L., Hahne, M., Frei, K., Fontana, A., and Tschopp, J. (1998) Conversion of membranebound Fas(CD95) ligand to its soluble form is associated with downregulation of its proapoptotic activity and loss of liver toxicity, J. Exp. Med. 187, 1205-13.

29. Holler, N., Tardivel, A., Kovacsovics-Bankowski, M., Hertig, S., Gaide, O., Martinon, F., Tinel, A., Deperthes, D., Calderara, S., Schulthess, T., Engel, J., Schneider, P., and Tschopp, J. (2003) Two adjacent trimeric Fas ligands are required for Fas signaling and formation of a death-inducing signaling complex, Mol. Cell. Biol. 23, 1428-40.

BI0516850 